\title{
T. GOLD
}

Space Science Building, Cornell University, Ithaca, N.Y. 14850, U.S.A.

The basic idea that pulsars may be the origin of cosmic rays arises when one realizes that they meet some of the criteria, perhaps not all as yet, for an origin theory of cosmic rays. Those criteria are: (1) there must be some possible mechanism that one can recognize for the acceleration of particles; and the pulsars have given us a very clear hint that particles are accelerated to at least the medium range of energies of cosmic rays $-10^{13} \mathrm{eV}$. (2) The objects that are thought responsible for cosmic rays must have enough total energy available to them to produce the entire cosmic ray beam in the Galaxy. I will come back to that, but it is clear that the total number of pulsars that one might expect in the Galaxy can indeed produce an adequate supply and even a good margin above that, if one makes the estimate on the basis of one plausible set of assumptions. (3) One would like of course that the mechanism proposed should generate the right spectrum; but there we as yet know too little. We do not understand what the energy spectrum of particles accelerated in pulsars should be, and we cannot as yet make any conclusive statement as to whether this spectrum can or cannot match the observed cosmic ray spectrum in the Galaxy. There is no reason for thinking otherwise, but there is no positive evidence in favor at the moment.

Then we have (4) the problem that the process selected must generate the correct composition. There we have the problem that we have just discussed, which is certainly a difficult one; I don't know that it is completely clear what type of particles you must expect to come from pulsars; how much of the material accelerated in the vicinity of the pulsar is material that is being cycled into it at the time and going out again; and how much is material which is preferentially sucked out of the surface, perhaps by reason of a high charge-to-mass ratio.

Then (5) the theory of origin of cosmic rays must be able to account for the observed degree of isotropy. There, as is known, even quite a weak but generally turbulent magnetic field in the Galaxy will of course suffice to isotropize particles coming from any stellar type of source distributed like the stars in the Galaxy, or like supernova sources. In that regard the pulsars would be adequately widely distributed if they were a common consequence of supernova explosions.

Now what can we say about the acceleration mechanism that occurs in the vicinity of pulsars? Firstly, we can say something about the energy density that is being radiated, and it is enormously high. The kind of figures that one comes to, as you have probably heard, amount to a brightness temperature at the source of the order of $10^{30}$ or $10^{31} \mathrm{deg}$, meaning that one is certainly dealing with collective phenomena on a very large scale; but also meaning that one is going to be very strained to account for this without each of the particles having a high relativistic factor, a high gamma.

We can get the order of magnitude of the gamma of the particles responsible for 
the radio emission by considering the bea mwidth that they radiate. We have to suppose that indeed pulsars are rotating beacons, so that the pulse length that one sees depends on the beamwidth that is sweeping around. One knows the rotation speed and one knows the pulse length, and one then observes the shortest pulses that can be seen. Therefore one knows the narrowest beamwidth of the source. That beamwidth is of course related to gamma if it is derived from the beaming of hyperrelativistic particles. Have we any reason for saying that the beamwidth is so derived rather than by some other antenna structure? Indeed we do, and it is the following - that we observe many pulsars over a great interval of frequency. In the case of the Crab pulsar, between optical radiation or X-ray radiation even, and the higher of the radio frequencies, $400 \mathrm{Mc}$, over the whole of that range the pulse length of individual pulses changes only very little; they get a little bit wider in the radio band, but not much. Over a wide range of frequencies the widening is very small, and that is after all very remarkable because if you attempted to do that with any kind of an antenna it would be a very artificial device. You would have to make a carefully compensated and contrived gadget. The one way that we do know for obtaining a roughly constant beamwidth, independent of emitted frequency, is when the high gamma of the radiating particles enforces a narrow beamwidth, and the total beamwidth of the beacon is given by the angle of the sector that contributes to the pulse. Individual pulses in the Crab pulsar are $100 \mu \mathrm{sec}$ or less in duration. To make such narrow pulses means that the source must make such narrow beams and that would imply a gamma of several hundred. One could suppose that one is only concerned with wider pulses and that these very short pulses are indeed time variable phenomena rather than due to narrow beaming (although I personally do not think so). One is still left with a gamma of more than a hundred.

We have another interesting piece of information that tells us something about the way in which the particles move in the vicinity of the pulsar, and also gives us a hint of the maximum particle energy to which a pulsar accelerates individual charges. I am referring to the famous 'march of the subpulses'. The phenomenon was first pointed out by Drake, that successive pulses have a subpulse in them that progressively gets to earlier phases within the pulse. Sometimes those subpulses are quite narrow. One must think out what that means in the actual three dimensional space in the vicinity of the pulsar. It means that there must be some locus of intense radiation associated with a bunch of relativistic particles which takes approximately one rotation period to move once around, that differs from one rotation period only by that small amount. So, if there is a bunch of particles whose motion is relativistic and they take about one period to go around, then they are somewhere in the vicinity of what $I$ have called the 'velocity of light cylinder'. It is very hard to accommodate these particles in any other place because if one puts them much further in, then they will have to make some path which quite fortuitously takes almost exactly one rotation to go around. If one puts them further out than the velocity of light cylinder, then they cannot get around in one rotation period because they would have to go faster than light to do so. So the fact that we have so clearly short, sharp pulses, repeating with 
only a small change in phase relative to the main envelope of the pulse, is an indication that indeed the radiation process is connected with the vicinity of the velocity of light cylinder - that is, the cylinder in space at which the rotation speed of the neutron star would be equal to the speed of light. Now if that is so, we can then make estimates (and many people have done) of the kind of particle energies that one can maintain in that location. It becomes clear that with the kind of fieldstrengths that one normally discusses (and also for many reasons other than fieldstrength) one would be limited to energies of about $10^{15} \mathrm{eV}$. Heavy particles may have somewhat higher particle energy, and if you stretch everything you could make $10^{17} \mathrm{eV}$ particles come from young, fast rotating pulsars.

So that makes a clear case that even in this location for the acceleration of the particles - the most favorable place in which to accelerate them - one cannot get to the peak energies of cosmic rays that have been observed, and so therefore I regret that I cannot propose that the pulsars are responsible for the entire range of energies of cosmic rays. On the other hand, if they were responsible for everything in excess of $10^{8}$ and up to $10^{15} \mathrm{eV}$, they would be responsible for more than $95 \%$ of the energy of the cosmic rays. The source of the high energy cosmic rays would be one whose mean energy requirement is very small, and it might therefore well be an extragalactic process that does not show itself at close range and therefore is much harder for us to discern.

The coincidence in the Crab Nebula that the energy put out by the particles would just fit the energy requirement to make the Crab Nebula shine, calculated on the basis that the drag on the neutron star that gives the deceleration is the main drag, is of course a very striking one. That is the famous $10^{38} \mathrm{erg}$ per sec, which is what one needs to make the Crab shine and what one needs to supply currently, as electrons, to the Crab shell; it is also the amount of energy that is abstracted from a neutron star that slows down by the observed amount and possesses the approximate value for the moment of inertia that neutron stars are thought to have. That coincidence I think must be taken seriously, and we must therefore think that at least in some circumstances, such as the ones in the Crab, fast particles are a major drain on rotational energy.

The rotational energy of a fresh neutron star might be generally of the order of $10^{52} \mathrm{erg}$. It is a very large amount, the same order of magnitude as the total nuclear energy that the star possessed before. If the losses of this rotational energy were dominated in the early phases by conversion into fast particles, as is suggested by the fact that we see the youngest pulsar evidently losing its energy predominantly in that way, then of course that would favor the pulsar cosmic ray origin. Almost all the energy of rotation is lost long before the object has got to the present stage of the Crab, because the Crab now has only $10^{49}$ or $10^{48}$ erg of rotational energy, and it might well have started with $10^{52}$. So therefore what happens to the rotational energy after the stage of the Crab Nebula pulsar is not terribly important perhaps to the whole discussion of the origin of comsic rays. It is the very early phases that count, since that is where the main energy is residing. And so, if it were true that the early 
pulsar loses the energy predominantly as fast particles then it would be good for the pulsar origin of cosmic rays. We would very much like to see another young pulsar so that we have more than one example of this being the case. It appears that for the old pulsars the electromagnetic radiation comes close to absorbing the entire drag, and therefore it does not look as if the particle loss of energy is the dominant component there.

If one takes favorable figures for the pulsars and supposes that the early loss is into particles, and that they are accelerated by dragging on the field and therefore draw on the rotational energy of the star, one might get on the order of $10^{52}$ erg from each supernova that results in a pulsar. If one had 1 per $100 \mathrm{yr}$ of those in the Galaxy, that would give $3 \times 10^{42}$ erg per sec as the energy supplied into the whole cosmic ray beam of the Galaxy. The requirements that the cosmic ray physicists have put on this figure is that with a reasonably high degree of magnetic confinement to the Galaxy they desire about $10^{41}$ erg per sec to be supplied. So we have a factor of 30 in hand, which one would like to use in part, of course, to make the galactic confinement of cosmic rays less perfect, but which one can also use in part to make the rate of supernovae making pulsars less than unity. It may well be that many supernovae are too massive to become pulsars. The question of the proportion of supernovae that one sees as pulsars can be discussed further. If the beams are narrow, then if just one beam existed around the pulsar of course there is only a small probability of seeing any particular supernova that has become a pulsar. The statistical evidence one can deduce from the observations is that perbaps of the order of 1 in 10 of the pulsars have a beam in any one arbitrarily chosen direction. The order of magnitude of 1 in 10 gives various consistencies in the statistics, and with that figure one can suppose that a reasonable fraction of supernovae do become pulsars, and of those in turn we only see of the order of a tenth.

So in summing up, some of the considerations look very favorable for pulsars as the origin of cosmic rays. We have seen for the first time a place where acceleration is really happening, and really happening in a big way, with large amounts of energy available and with undoubtedly high particle energies being produced there. We have seen, in other words, a cosmic particle accelerator at work. That cannot be doubted anymore. Whether we can deduce the details of the circumstances in the vicinity of the pulsars well enough to understand the composition and the spectrum problems - that is a matter that $\mathrm{I}$ cannot at the moment predict.

\section{References}

Drake, F. D. and Craft, H. D., Jr.: 1968, Nature 220, 5164.

Gold, T.: 1969, Nature 223, 5202.

Gold, T.: 1969, Nature 221, 5175.

Gold, T.: 1971, Proc. of the XI International Conf. on Cosmic Rays, Publ. Central Res. Inst. of Phys. of the Hungarian Academy of Sciences, Budapest, 1971. 